\title{
revisão de literatura Consciência do déficit na doença de Alzheimer: diferenças entre os domínios cognitivo e funcional
}

Awareness of deficit in Alzheimer's disease: the differences

between the cognitive and functional domain

Tatiana Belfort', Maria Fernanda Barroso de Sousa', Raquel Luiza Santos', Marcela Lima', Bianca Torres', Rachel Dias', Marcia Cristina Nascimento Dourado ${ }^{1}$

\section{RESUMO}

Objetivos: Revisar sistematicamente desenhos de estudo, instrumentos de avaliação e fatores relacionados ao comprometimento dos domínios cognitivo e funcional da consciência do déficit em pessoas com doença de Alzheimer (DA). Método: Pesquisa nas bases de dados PubMed e ISI de estudos sobre consciência do déficit na DA publicados entre 2008 e 2013. As palavras-chave utilizadas foram: "dementia", "Alzheimer", "awareness", "awareness of memory" e "awareness of functioning", "deficits", "cognition". Resultados: Os 10 artigos selecionados utilizaram os conceitos "falta de consciência do déficit", "anosognosia", "insight", "falta de consciência das dificuldades cognitivas" e "consciência limitada das deficiências". A etiologia do comprometimento da consciência do déficit foi relacionada a fatores biológicos como gravidade clínica da doença, associações neuroanatômicas, alterações neuropsiquiátricas e fatores psicológicos e sociais. O desenho de estudo mais utilizado foi o corte transversal. No domínio cognitivo, a memória e as funções executivas foram as duas principais funções investigadas. No domínio funcional, foram encontradas associações com a disfunção executiva, declínio da interação social, depressão e a influência do desempenho ocupacional. Conclusões: As distintas hipóteses etiológicas, a operacionalização variável do conceito e a falta de instrumentos de avaliação padronizados impossibilitam a obtenção de resultados homogêneos. Essas dificuldades comprometem a compreensão e a investigação dos domínios cognitivo e funcional.

\section{ABSTRACT}

Objectives: To systematically review the study designs, assessment measurements and factors related to the impairment of cognitive and functional domains of awareness of deficits in older adults with Alzheimer's disease (AD). Method: Search at PubMed and ISI databases about studies on awareness of deficit in AD, published from 2008 to 2013. We used the keywords "Dementia", "Alzheimer", "Awareness", "Awareness of memory" and "Awareness of Functioning", "deficits", "cognition". Results: The ten articles selected used the concepts of "lack of awareness of deficits", "anosognosia", "insight", "lack of awareness of cognitive difficulties" and "limited awareness of disabilities". The etiology of impaired awareness of deficit

1 Universidade Federal do Rio de Janeiro (UFRJ), Instituto de Psiquiatria (IPUB), Centro para Doença de Alzheimer e outros Transtornos Mentais na Velhice. 


\section{Keywords}

Dementia, anosognosia, awareness of deficit, cognitive functions, activities of daily living. was related to biological factors such as clinical severity, neuroanatomical associations, neuropsychiatric and psychological and social factors. The cross-sectional study design was the most used. In the cognitive domain, memory and executive functions were the two main functions investigated. In the functional domain, we found associations with executive dysfunction, social interaction decline, depression and influence of occupational performance.

Conclusions: Homogeneous results are hindered by several etiological hypotheses, different terms' operationalization and lack of standardized assessment instruments. It affects the knowledge and investigation about cognitive and functional domains.

\section{INTRODUÇÃO}

O comprometimento da consciência do déficit, também denominado como anosognosia ${ }^{1-4}$, é um fenômeno comumente associado às inúmeras alterações presentes na doença de Alzheimer (DA). A consciência do déficit é definida como a capacidade de perceber em si e/ou nas atividades da vida diária (AVD) alterações causadas por déficits relacionados ao processo de adoecimento ${ }^{5}$.

Esse fenômeno pode ser explicado por dois modelos etiológicos relacionados a fatores orgânicos e psicossociais ${ }^{6}$. 0 primeiro modelo investiga o comprometimento da consciência do déficit sob a influência de fatores como a gravidade clínica da doença, a disfunção do lobo frontal e a presença de sintomas psicológicos e do comportamento na demência (SPCD) ${ }^{7}$, enquanto o modelo relacionado aos fatores psicossociais baseia-se nas características da personalidade, mecanismos de defesa, crenças sobre saúde/doença, diagnóstico tardio, falhas ou dificuldades em iniciar o tratamento, conflito com os familiares/cuidadores e o contexto da avaliação ${ }^{8,9}$.

A consciência do déficit é um constructo complexo, que possui variadas manifestações clínicas ${ }^{10-12}$, pois pode ser expressa por meio de uma simples percepção ou de uma sofisticada consciência de si e do processo de adoecimento 7 . Esse constructo abrange áreas diversas como as relações sociais, familiares, afetivas e as atividades de vida diária9 ${ }^{\text {. As- }}$ sim sendo, o comprometimento da consciência do déficit pode apresentar variados graus de acometimento (normal, parcial, ausente, intermitente e flutuante) ${ }^{13}$, com alterações em diversos domínios, ou seja, alguns domínios podem estar comprometidos e outros preservados ${ }^{14,13}$. Dessa forma, o nível de comprometimento da consciência do déficit está relacionado com o prejuízo em cada domínio ${ }^{15}$.

Os domínios cognitivo e funcional são os mais comumente estudados, em especial nas pessoas com DA ${ }^{16}$. Contudo, estudos recentes demonstram que existem controvérsias relacionadas aos fatores responsáveis pelo comprometimento da consciência do déficit nesses domínios 5,15,17,18. O estudo sobre a consciência do déficit é clinicamente relevante, pois está relacionado tanto com a avaliação da autonomia das pessoas com DA e as intervenções necessárias para sua proteção quanto com a real capacidade dessas pessoas em compreender e colaborar com as estratégias de reabilitação e tratamento propostas.
Tendo em vista a complexidade do fenômeno, esta revisão sistemática tem como objetivo examinar comparativamente os desenhos de estudo, instrumentos de avaliação e fatores relacionados ao comprometimento dos domínios cognitivo e funcional da consciência do déficit em pessoas com DA.

\section{MÉTODO}

Foi realizada uma busca nas bases Web of Knowledge Cross Search (Thomson Scientific/ISI Web Services) e PubMed, procurando artigos de janeiro de 2008 a janeiro de 2013 em inglês que descrevessem o comprometimento da consciência do déficit em pessoas com DA. As palavras-chave utilizadas foram: "dementia", "Alzheimer", "awareness", "awareness of memory", "awareness of functioning", "deficits", "cognition", com as combinações "dementia AND awareness AND deficits", "Alzheimer AND awareness of memory AND cognition", "Alzheimer AND awareness of functioning AND cognition". Foram adotados os seguintes limites: humans, all adults: +65 years.

Foram incluídos estudos transversais, longitudinais, randomizados ou não randomizados, com ou sem grupo controle, com pessoas com demência senil e pré-senil. Foram excluídos artigos de revisão, estudos que avaliaram somente intervenção farmacológica ou não farmacológica ou que associaram consciência do déficit a traumatismo cranioencefálico, condições metabólicas e qualquer outra etiologia de demência.

Inicialmente, todos os resumos selecionados foram lidos por dois avaliadores. Os avaliadores agruparam de forma independente o objetivo, desenho do estudo, metodologia, instrumentos utilizados e resultados relacionados à multidimensionalidade da consciência do déficit na DA. Os resumos aprovados pelos dois avaliadores eram incluídos na revisão, e os que apresentassem discordância eram submetidos a um terceiro avaliador.

\section{RESULTADOS}

Foram encontrados 200 artigos, sendo 129 na base ISI e 71 na PubMed. Retiradas as referências constantes em mais de uma 
base, foram selecionados 10 artigos. Os artigos excluídos desta revisão $(N=109)$ foram agrupados em categorias, como desenho do estudo, participantes, intervenções, outros fatores relacionados ao comprometimento na demência e avaliação.

Foram utilizados conceitos como falta de consciência do déficit $(n=7)^{16,19-24}$, anosognosia $(n=4)^{19,20,23,25}$, insight $(n=$ 2) ${ }^{19,25}$, falta de consciência das dificuldades cognitivas ( $n=$ 1) $)^{10}$ e limitada consciência das deficiências $(n=1)^{26}$.

A etiologia do comprometimento da consciência do déficit foi relacionada a fatores biológicos como associações neuroanatômicas $(n=2)^{16,23}$, alterações neuropsiquiátricas ( $n$ $=3)^{10,16,20}$ e a gravidade clínica da doença, também investigada pelo modelo psicossocial $(n=9)^{10,16,19,20-25}$. Os artigos que relacionaram o comprometimento da consciência do déficit aos fatores psicossociais $(n=4)^{21,22,24,26}$ abordaram aspectos como a influência de fatores psicológicos e sociais $(n=1)^{21}$, 0 impacto nas AVD $(n=2)^{22,26}$ e o valor prognóstico da avaliação global do cuidado na avaliação médica $(n=1)^{24}$.

Na análise dos domínios da consciência do déficit foram encontrados os domínios cognitivo e funcional $(n=10)^{10,16,19-26}$, comportamental $(n=5)^{16,19-22}$, emocional e social $(n=2)^{21,22}$.

No domínio cognitivo, a memória e as funções executivas foram as duas principais funções investigadas $(n=8)^{10,16,19-}$ 23,25, enquanto os demais artigos investigaram a cognição de forma global $(n=2)^{24,26}$. No domínio funcional foram encon- tradas associações com as funções cognitivas $(n=5)^{16,20,24-26}$, com destaque para a disfunção executiva $(n=2)^{16,20}$, o declínio da interação social $(n=2)^{21,22}$, o impacto da depressão ( $n$ $=2)^{22,26}$ e a influência do desempenho ocupacional $(n=1)^{26}$.

O desenho metodológico mais utilizado foi o corte transversal $(n=7)^{16,19,20,23-25,26}$, com apenas três estudos utilizando o método longitudinal. Não foram encontrados estudos randomizados.

A consciência do déficit foi avaliada de três formas distintas: questionário de discrepância entre o relato do paciente e de seu cuidador $(n=8)^{10,16,19-23,25}$, questionário de discrepância entre o relato do paciente e seu desempenho em testes objetivos $(n=2)^{24,26}$ e entrevista clínica $(n=1)^{24}$.

Os principais instrumentos utilizados avaliaram a consciência do déficit em seus aspectos globais, sendo eles: Anosognosia Questionnaire-Dementia (AQ-D) $(n=3)^{16,19,20}$, Clinical Insight Rating $(\mathrm{CIR})(\mathrm{n}=1)^{25}$, Cognitive Difficulties Scale $(\mathrm{CDS})(\mathrm{n}=1)^{24}$, Measurement of Anosognosia Instrument (MAI) $(n=1)^{23}$ e Assessment of Awareness of Disability (AAD) $(n=1)^{26}$, contudo alguns instrumentos avaliam os aspectos globais a partir do somatório dos domínios específicos, como é o caso do AQ-D. Para a avaliação de domínios específicos, foram utilizados: o Functional Activities Questionnaire (FAQ) $(n=2)^{21,22}$, o Everyday Memory Checklist (EMC) $(n=1)^{10}$, o Memory Functioning Scale (MFS) $(n=$ $2)^{21,22}$ e o Socio-Emotional Questionnaire (SEQ) $(n=2)^{21,22}$.

Tabela 1. Seleção dos estudos para revisão

\begin{tabular}{|c|c|c|}
\hline Domínios & Critérios de exclusão & Excluídos \\
\hline Participantes & $\begin{array}{c}\mathrm{N}=109 \\
\downarrow \\
\text { Outros transtornos } \\
\text { Outras síndromes neurodegenerativas } \\
\text { Processos neurais e biológicos } \\
\text { Idosos saudáveis } \\
\downarrow \\
\mathrm{N}=58\end{array}$ & $\begin{array}{l}18 \\
16 \\
15 \\
2\end{array}$ \\
\hline Outros fatores relacionados ao comprometimento na demência & $\begin{array}{l}\text { Fatores neuropsicológicos } \\
\text { Consciência: domínio cognitivo } \\
\text { Fatores psicossociais } \\
\text { Déficits olfativos } \\
\text { Consciência: outros domínios } \\
\downarrow \\
\mathrm{N}=30\end{array}$ & $\begin{array}{l}9 \\
7 \\
7 \\
1 \\
4\end{array}$ \\
\hline Desenho do estudo & $\begin{array}{c}\text { Revisão } \\
\text { Pesquisa com animais } \\
\text { Prática clínica } \\
\downarrow \\
N=15\end{array}$ & $\begin{array}{c}12 \\
1 \\
2\end{array}$ \\
\hline Intervenções & $\begin{array}{c}\text { Não farmacológicas } \\
\text { Farmacológicas } \\
\text { Cuidadores - profissionais de saúde } \\
\downarrow \\
N=7\end{array}$ & $\begin{array}{l}4 \\
2 \\
2\end{array}$ \\
\hline Avaliação & $\begin{array}{c}\text { Propriedades psicométricas } \\
\text { Direção de veículo } \\
\downarrow \\
N=00\end{array}$ & $\begin{array}{l}6 \\
1\end{array}$ \\
\hline
\end{tabular}


Tabela 2. Tabela de artigos com os domínios investigados

\begin{tabular}{|c|c|c|c|c|c|c|c|}
\hline Autor/Ano & Termo utilizado & Hipótese etiológica & Amostra & $\begin{array}{l}\text { Desenho do } \\
\text { estudo }\end{array}$ & $\begin{array}{l}\text { Domínios } \\
\text { investigados }\end{array}$ & $\begin{array}{l}\text { Avaliação } \\
\text { Consciência }\end{array}$ & Resultados \\
\hline $\begin{array}{l}\text { Amanzio } \\
\text { et al. } \\
(2013)\end{array}$ & $\begin{array}{l}\text { Falta de } \\
\text { consciência do } \\
\text { déficit }\end{array}$ & $\begin{array}{l}\text { Fator orgânico: } \\
\text { associação } \\
\text { neuroanatômica, } \\
\text { gravidade clínica da } \\
\text { doença, alterações } \\
\text { neuropsiquiátricas }\end{array}$ & $\begin{array}{l}117 \text { DA inicial } \\
117 \text { cuidadores }\end{array}$ & Transversal & $\begin{array}{l}\text { Cognitivo } \\
\text { Comportamental } \\
\text { Funcional }\end{array}$ & $\begin{array}{l}\text { AQ-D } \\
\text { (questionário de } \\
\text { discrepância paciente- } \\
\text { cuidador) }\end{array}$ & $\begin{array}{l}\text { Comprometimento das funções executivas } \\
\text { importante para a consciência das atividades } \\
\text { instrumentais da vida diária } \\
\text { Disfunção do lobo frontal associada à redução } \\
\text { da consciência dos déficits } \\
\text { Desinibição e apatia são indicadores da redução } \\
\text { da consciência comportamental em pacientes } \\
\text { com DA } \\
\text { Não há relação linear entre a consciência dos } \\
\text { déficits e a progressão da gravidade da doença }\end{array}$ \\
\hline $\begin{array}{l}\text { Gallo } \\
\text { et al. } \\
(2012)\end{array}$ & $\begin{array}{l}\text { Anosognosia } \\
\text { Falta de } \\
\text { consciência do } \\
\text { déficit } \\
\text { Insight }\end{array}$ & $\begin{array}{l}\text { Fator orgânico: } \\
\text { gravidade clínica da } \\
\text { doença, alterações } \\
\text { neuropsiquiátricas }\end{array}$ & $\begin{array}{l}18 \mathrm{DA} \\
18 \mathrm{CN} \\
18 \text { cuidadores }\end{array}$ & Transversal & $\begin{array}{l}\text { Cognitivo } \\
\text { Comportamental } \\
\text { Funcional }\end{array}$ & $\begin{array}{l}\text { AQ-D (questionário de } \\
\text { discrepância paciente- } \\
\text { cuidador) }\end{array}$ & $\begin{array}{l}\text { A consciência do déficit de memória é } \\
\text { preservada nos estágios iniciais da DA } \\
\text { Melhorar as atividades metacognitivas pode } \\
\text { atenuar as consequências psicológicas e } \\
\text { comportamentais na DA }\end{array}$ \\
\hline $\begin{array}{l}\text { Maki } \\
\text { et al. } \\
(2012)\end{array}$ & $\begin{array}{l}\text { Falta de } \\
\text { consciência do } \\
\text { déficit } \\
\text { Anosognosia }\end{array}$ & $\begin{array}{l}\text { Fator orgânico: } \\
\text { gravidade clínica da } \\
\text { doença, alterações } \\
\text { neuropsiquiátricas }\end{array}$ & $\begin{array}{l}12 \mathrm{DCL} \\
23 \mathrm{DA} \text { inicial } \\
18 \mathrm{DA} \\
\text { moderado } \\
53 \text { cuidadores }\end{array}$ & Transversal & $\begin{array}{l}\text { Cognitivo } \\
\text { Comportamental } \\
\text { Funcional }\end{array}$ & $\begin{array}{l}\text { AQ-D (questionário de } \\
\text { discrepância paciente- } \\
\text { cuidador) }\end{array}$ & $\begin{array}{l}\text { A consciência do déficit permanece até } 0 \\
\text { estágio moderado } \\
\text { Redução da consciência dos déficits nas } \\
\text { atividades que envolvem o funcionamento } \\
\text { executivo }\end{array}$ \\
\hline $\begin{array}{l}\text { Clare } \\
\text { et al. } \\
(2012)\end{array}$ & $\begin{array}{l}\text { Falta de } \\
\text { consciência do } \\
\text { déficit }\end{array}$ & Fator psicossocial & $\begin{array}{l}51 \mathrm{DA} \\
30 \mathrm{DV} \\
20 \mathrm{DM} \\
101 \text { cuidadores }\end{array}$ & Longitudinal & $\begin{array}{l}\text { Cognitivo } \\
\text { Comportamental } \\
\text { Funcional } \\
\text { Emocional } \\
\text { Social }\end{array}$ & $\begin{array}{l}\text { MFS } \\
\text { SEQ } \\
\text { FAQ (questionários de } \\
\text { discrepância paciente- } \\
\text { cuidador) }\end{array}$ & $\begin{array}{l}\text { A alteração da consciência do déficit não está } \\
\text { diretamente relacionada com a progressão e a } \\
\text { gravidade da demência } \\
\text { Dificuldades nas atividades da vida diária são } \\
\text { mais evidentes do que as alterações na memória } \\
\text { ou reconhecimento socioemocional }\end{array}$ \\
\hline $\begin{array}{l}\text { Clare } \\
\text { et al. } \\
(2012)\end{array}$ & $\begin{array}{l}\text { Falta de } \\
\text { consciência do } \\
\text { déficit }\end{array}$ & Fator psicossocial & $\begin{array}{l}101 \text { PCD no } \\
\text { estágio inicial } \\
101 \text { cuidadores }\end{array}$ & Longitudinal & $\begin{array}{l}\text { Cognitivo } \\
\text { Comportamental } \\
\text { Funcional } \\
\text { Emocional } \\
\text { Social }\end{array}$ & $\begin{array}{l}\text { MFS } \\
\text { FAQ } \\
\text { SEQ (questionários de } \\
\text { discrepância paciente- } \\
\text { cuidador) }\end{array}$ & $\begin{array}{l}\text { Fatores sociais e psicológicos influenciam os } \\
\text { escores que avaliam a consciência do déficit, } \\
\text { assim como fatores neuropsicológicose } \\
\text { relacionados à doença }\end{array}$ \\
\hline $\begin{array}{l}\text { Ohman } \\
\text { et al. } \\
\text { (2011) }\end{array}$ & $\begin{array}{l}\text { Limitada } \\
\text { consciência das } \\
\text { deficiências }\end{array}$ & Fator psicossocial & $\begin{array}{l}8 \mathrm{DCL} \\
18 \mathrm{DA} \\
5 \mathrm{DV} \\
4 \text { outras } \\
\text { demências }\end{array}$ & Transversal & $\begin{array}{l}\text { Cognitivo } \\
\text { Funcional }\end{array}$ & $\begin{array}{l}\text { AAD (discrepância entre } \\
\text { o relato do paciente e } 0 \\
\text { desempenho no teste) }\end{array}$ & $\begin{array}{l}0 \text { aumento da consciência das deficiências } \\
\text { relacionado ao aumento da limitação no } \\
\text { desempenho das AVD } \\
\text { Depressão na DA tem impacto negativo nas AVD }\end{array}$ \\
\hline $\begin{array}{l}\text { Stewart } \\
\text { et al. } \\
(2010)\end{array}$ & $\begin{array}{l}\text { Falta de } \\
\text { consciência do } \\
\text { déficit } \\
\text { Anosognosia }\end{array}$ & $\begin{array}{l}\text { Fator orgânico: } \\
\text { gravidade clínica da } \\
\text { doença e associação } \\
\text { neuroanatômica }\end{array}$ & $\begin{array}{l}23 \text { DA provável } \\
\text { inicial a } \\
\text { moderado } \\
30 \mathrm{CN}\end{array}$ & Transversal & $\begin{array}{l}\text { Cognitivo } \\
\text { Funcional }\end{array}$ & $\begin{array}{l}\text { MAI (questionário de } \\
\text { discrepância paciente- } \\
\text { cuidador) }\end{array}$ & $\begin{array}{l}\text { Relação positiva entre } 0 \text { aumento da } \\
\text { anosognosia e a progressão da doença } \\
\text { Nos estágios iniciais da DA o comprometimento } \\
\text { da consciência do déficit e as dificuldades de } \\
\text { memória estariam relacionados a alterações da } \\
\text { estrutura temporal medial }\end{array}$ \\
\hline $\begin{array}{l}\text { Okonkwo } \\
\text { et al. } \\
(2010)\end{array}$ & $\begin{array}{l}\text { Falta de } \\
\text { consciência do } \\
\text { déficit }\end{array}$ & $\begin{array}{l}\text { Fator } \\
\text { psicossocial }\end{array}$ & $\begin{array}{l}108 \text { pacientes } \\
108 \text { cuidadores }\end{array}$ & Transversal & $\begin{array}{l}\text { Cognitivo } \\
\text { Funcional }\end{array}$ & $\begin{array}{l}\text { CDS (entrevista clínica; } \\
\text { discrepância paciente- } \\
\text { cuidador; } \\
\text { discrepância entre } 0 \\
\text { relato do paciente e } 0 \\
\text { desempenho no teste) }\end{array}$ & $\begin{array}{l}\text { A avaliação global do cuidador sobre a } \\
\text { consciência do déficit é um preditor significativo } \\
\text { na avaliação médica } \\
\text { A discrepância entre os dados do cuidador e do } \\
\text { paciente com relação às dificuldades cognitivas } \\
\text { não foi significativa } \\
0 \text { comprometimento da consciência do déficit } \\
\text { piora com a progressão da doença }\end{array}$ \\
\hline $\begin{array}{l}\text { Leicht } \\
\text { et al. } \\
(2010)\end{array}$ & $\begin{array}{l}\text { Anosognosia } \\
\text { Insight }\end{array}$ & $\begin{array}{l}\text { Fator orgânico: } \\
\text { gravidade clínica da } \\
\text { doença }\end{array}$ & $\begin{array}{l}32 \text { DA inicial e } \\
\text { moderado } \\
32 \text { cuidadores }\end{array}$ & Transversal & $\begin{array}{l}\text { Cognitivo } \\
\text { Funcional }\end{array}$ & $\begin{array}{l}\text { CIR (discrepância } \\
\text { paciente-cuidador) }\end{array}$ & $\begin{array}{l}0 \text { aumento dos déficits na progressão da DA } \\
\text { compromete a avaliação da anosognosia } \\
\text { A anosognosia aumenta com a gravidade da } \\
\text { demência }\end{array}$ \\
\hline $\begin{array}{l}\text { Akai } \\
\text { et al. } \\
(2009)\end{array}$ & $\begin{array}{l}\text { Falta de } \\
\text { consciência das } \\
\text { dificuldades } \\
\text { cognitivas }\end{array}$ & $\begin{array}{l}\text { Fator orgânico: } \\
\text { gravidade clínica da } \\
\text { doença e alterações } \\
\text { neuropsiquiátricas }\end{array}$ & 62 DA inicial & Longitudinal & $\begin{array}{l}\text { Cognitivo } \\
\text { Funcional }\end{array}$ & $\begin{array}{l}\text { EMC (discrepância } \\
\text { paciente-cuidador) }\end{array}$ & $\begin{array}{l}0 \text { prejuízo da consciência do déficit progride } \\
\text { com o declínio cognitivo } \\
0 \text { comprometimento da consciência do déficit } \\
\text { de memória foi relacionado positivamente com } \\
\text { alterações neuropsiquiátricas }\end{array}$ \\
\hline
\end{tabular}

Amostra: CN: controle normal; DA: doença de Alzheimer; DCL: declínio cognitivo leve; DM: demência mista; DV: demência vascular; N: não possui controle; PCD: pessoas com demência. Instrumentos: AAD: Assessment of Awareness of Disability; AQ-D: Awareness of Deficits Questionnaire-Dementia Scale; CDS: Cognitive Difficulties Scale; CIR: Clinical Insight Rating; EMC: Everyday Memory Checklist; FAQ: Functional Activities Questionnaire; MAI: Measurement of Anosognosia Instrument; MFS: Memory Functioning Scale; SEQ: Socio-Emotional Questionnaire. Resultados: AVD: atividades da vida diária; SPCD: sintomas psicológicos e comportamentais na demência. 


\section{DISCUSSÃO}

\section{Operacionalização do conceito e hipóteses etiológicas para o comprometimento da consciência do déficit}

Os termos "falta de consciência do déficit"16,19-24, "anosognosia"19,20,23,25, "insight"19,25, "falta de consciência das dificuldades cognitivas"10 e "limitada consciência das deficiências"26 são usados como sinônimos para definir e pesquisar tanto aspectos mais limitados como a cognição quanto os mais extensos como as deficiências em geral. Podemos, também, considerar que a utilização de conceitos com significados específicos como anosognosia ou vagos como insight resulta em falta de consenso teórico e consequentes diferenças metodológicas, como a falta de instrumentos padronizados na avaliação, além da heterogeneidade das amostras na área. Portanto, é necessária melhor delimitação conceitual que facilite a operacionalização das hipóteses e objetivos propostos nos estudos sobre a consciência do déficit na DA.

Nos estudos selecionados observa-se que a maioria associa o comprometimento da consciência do déficit à gravidade clínica da doençaa,16,19-21,23-26. Entretanto, enquanto alguns estudos encontraram uma relação linear $r^{10,20,23-25}$, outros se opuseram a esses resultados ${ }^{16,21}$. Clare et al. ${ }^{21}$ observaram que a consciência do déficit estaria comprometida desde os estágios iniciais da demência, mas haveria estabilização desse comprometimento nos estágios mais avançados da doença.

Nos estudos voltados para as associações neuroanatômicas, o comprometimento da consciência do déficit foi relacionado à disfunção do lobo frontal e à disfunção executiva em pessoas nos estágios iniciais da doença ${ }^{16}$. Entretanto, Stewart et al. ${ }^{23}$ contrariaram essa associação sugerindo que o comprometimento nos estágios iniciais estaria relacionado às alterações no lobo temporal medial, tendo em vista a intensa perda de células hipocampais e células neuronais no córtex perirrinal.

Existe consenso sobre a relação entre o comprometimento da consciência do déficit e as alterações neuropsiquiátricas ${ }^{10,16,19,20}$. A presença de alterações comportamentais, ainda que discretas, pode ser considerada como marcador em pessoas com comprometimento da consciência do déficit. A apatia e a desinibição foram as principais responsáveis pelo comprometimento do domínio comportamental da consciência do déficit nas pessoas com DA ${ }^{16}$.

Em oposição ao modelo biológico, criticado por reduzir o fenômeno a um déficit específico ou considerá-lo um sintoma independente nos indivíduos com demência, os estudos integrantes do modelo psicossocial sugerem que a consciência do déficit é um fenômeno complexo influenciado pela gravidade clínica da doença e por aspectos emocionais e comportamentais. Clare et al..$^{22}$ demonstraram a influência dos fatores sociais e psicológicos na avaliação da consciência do déficit, comparando-os aos fatores neuropsicológicos relacionados à doença. Altos níveis de ansiedade estariam associados a piores relatos de memória e reconhecimento social, enquanto a presença de depressão maior estaria mais relacionada a piores relatos funcionais ${ }^{22}$.

\section{Consciência do déficit cognitivo}

A maioria dos estudos sugeriu uma relação positiva entre a alteração do funcionamento cognitivo e o comprometimento da consciência do déficit ${ }^{10,16,19-25}$. Os principais domínios cognitivos investigados foram memória e funções executi$\operatorname{vas}^{10,16,19-23,25}$

A disfunção executiva é uma das alterações cognitivas presentes na demência e os componentes mais comumente prejudicados são o controle inibitório, a capacidade de automonitoramento e a flexibilidade cognitiva ${ }^{16}$. O prejuízo na flexibilidade cognitiva foi relacionado ao maior comprometimento da consciência do déficit, ao contrário das habilidades de planejamento, que não demonstraram ter relação tão estreita com esse fenômeno. A flexibilidade cognitiva pode ser entendida pela capacidade de monitorar as informações detectando erros e atualizando o mecanismo comparador do modelo de Agnew e Morris (1998)25, dessa forma a rigidez cognitiva reduz a capacidade de reconhecer os próprios erros e dificuldades ${ }^{16}$. Além disso, a flexibilidade cognitiva foi também associada ao reconhecimento das próprias percepções, comportamentos, sintomas e experiências relacionadas à doença e à presença de reações inusitadas quando comparadas ao funcionamento de uma pessoa saudável ${ }^{16}$. Maki et al. ${ }^{20}$ relataram estreita relação da metacognição com as funções executivas e observaram que o comprometimento das funções executivas pode ser anterior ao comprometimento mnemônico e de orientação.

Leicht et al..$^{25}$ se basearam no modelo neurocognitivo de consciência (Counscious Awareness System) proposto por Agnew e Morris (1998) para explicar as diferenças encontradas entre o autorrelato e o relato do cuidador. Quando as discrepâncias estão associadas ao comprometimento executivo, há dificuldade em detectar a diferença entre as experiências atuais e o conhecimento prévio (memória) (anosognosia executiva). Na associação com o comprometimento mnemônico são observadas dificuldades na atualização das autoavaliações relacionadas aos déficits de memória (anosognosia mnemônica $)^{25}$.

A consciência do déficit de memória pode estar preservada nos estágios iniciais da doença ${ }^{16,19,20}$, entretanto alterações na capacidade de reconhecimento de problemas cotidianos de memória sugerem que a relação entre metacognição local (monitoramento imediato do desempenho na tarefa cognitiva específica) e global (informações da me- 
mória de longo prazo que envolvem habilidades pessoais ou crenças de autoeficácia) pode estar associada a domínios específicos ${ }^{19}$. Maki et al. ${ }^{20}$ relataram que para os cuidadores a consciência preservada do déficit de memória e as dificuldades na escrita seriam as principais causas de ansiedade nas pessoas com DA nos estágios iniciais.

Akai et al. ${ }^{10}$ sugeriram que há correlação positiva entre delírios, apatia e agitação e o comprometimento da consciência do déficit de memória, enquanto, por outro lado, sintomas de depressão e ansiedade estariam correlacionados negativamente. Clare et al. ${ }^{21}$ demonstraram que o autoconceito seria um mecanismo de negação psicológico relevante para a memória e para o funcionamento social. Um bom autoconceito estaria associado a melhores relatos de memória e do funcionamento social, o que indicaria uma manutenção do estilo de enfrentamento e falta de atualização das mudanças recentes.

\section{Consciência do déficit funcional}

As alterações cognitivas na DA podem estar associadas, desde os estágios iniciais, ao prejuízo nas AVD ${ }^{16,20,24-26}$, que refletem principalmente em atividades como controle financeiro, práticas de hobbies e atividades domésticas ${ }^{20}$. As pessoas com DA são capazes de identificar as alterações na rotina, mas subestimam a gravidade quando comparadas ao grupo controle, demonstrando comprometimento da consciência do déficit funcional ${ }^{19}$.

A consciência do déficit seria, então, um fenômeno caracterizado pela especificidade dos domínios, que variam de acordo com os diferentes objetos (memória, AVD e funcionamento social). Pessoas com DA podem reconhecer mais facilmente dificuldades em um determinado domínio do que em outros, quer por serem mais evidentes ou por repercutirem de forma mais direta e incontestável na funcionalidade, como as AVD21. O aumento das dificuldades nas AVD parece estar relacionado com o comprometimento da consciência dos déficits, com piora no quadro quando associado à depressão ${ }^{26}$.

Ainda que o declínio nas AVD tenha sido observado tanto na perspectiva da pessoa com DA quanto do cuidador, as maiores discrepâncias entre os relatos foram observadas nessa área ${ }^{22,25}$. Na perspectiva das pessoas com DA, o comprometimento de atividades que envolvam a interação social e tarefas intelectuais relacionadas à comunicação, tais como dificuldade de nomeação, problemas com a escrita e por compreensão de textos, são os preditores de ansiedade nos estágios iniciais da doença ${ }^{20}$. Os autorrelatos mais positivos nas habilidades funcionais foram associados com maior escolaridade e melhores resultados para evocação, nomeação e fluência fonêmica, enquanto autorrelatos negativos foram associados com depressão e melhores resultados para fluência e conhecimento semân- tico ${ }^{21}$. Essa relação positiva entre autorrelato e habilidade funcional reflete a importância da escolaridade na investigação das AVD, pois uma melhor capacidade cognitiva pré-mórbida está relacionada à maior reserva cognitiva e consequentemente a menor comprometimento da consciência do déficit ${ }^{27}$.

\section{Metodologia e instrumentos: dificuldades na avaliação da consciência do déficit e seus domínios}

O desenho de estudo mais utilizado foi o corte transversal16,19,20,23-26, apesar das limitações metodológicas para o estudo de um fenômeno flutuante como a consciência do déficit. Os estudos longitudinais são os mais adequados, pois permitem tanto acompanhar a progressão do comprometimento dos diferentes domínios ao longo da doença quanto a relação com outras variáveis, como os declínios cognitivo e funcional ${ }^{10,21}$. O reduzido número de estudos longitudinais ${ }^{10,21,22}$, associado principalmente ao agravamento da doença e à dificuldade de acompanhar uma grande quantidade de pessoas, prejudica a observação das variações desse fenômeno.

O principal método de avaliação da consciência do déficit foi o questionário de discrepância entre os relatos de paciente e cuidador 10,16,19,20-23,25. Esse tipo de avaliação tem mostrado que as pessoas com DA, quando comparadas aos cuidadores, subestimam suas dificuldades de memória e nas suas atividades de vida diária. Uma limitação desse método de avaliação se refere à falta de imparcialidade no julgamento dos cuidadores. Estudos de comparação entre pacientes e cuidadores têm demonstrado que a avaliação deles se encontra intimamente associada à dinâmica familiar, à sobrecarga e aos sintomas depressivos do cuidador ${ }^{10,21-23,25}$. Assim, a avaliação dos cuidadores pode estar comprometida por dificuldades em lidar com a doença ou por não serem os informantes mais adequados sobre as alterações.

Outra crítica à análise da discrepância entre os relatos dos pacientes e cuidadores foi a relação de especificidade dos domínios, que pode influenciar a avaliação das pessoas com $D^{22}$. Okonkwo et al..$^{24}$ sugeriram que a discrepância entre as avaliações não necessariamente apontam um comprometimento da consciência do déficit.

Outros métodos utilizados foram o questionário de discrepância entre o relato do paciente e seu desempenho em testes objetivos ${ }^{24,26}$ e a entrevista clínica ${ }^{24}$. No entanto, em sua maioria, essas avaliações transformam um constructo contínuo em um fenômeno dicotômico analisado simplesmente pela sua presença ou ausência. A análise dos métodos de avaliação demonstra que a aplicação de diferentes instrumentos que oferecem apenas dados parciais pode ser um fator limitador para o entendimento da consciência do déficit. Uma alternativa seria a combinação entre os diferentes instrumentos de avaliação na tentativa de melhor entender a complexidade do fenômeno ${ }^{24}$. 


\section{CONCLUSÃO}

A consciência do déficit é a capacidade de perceber a presença de déficits cognitivos e/ou de prejuízos funcionais causados pela DA. Por ser um constructo multidimensional, o reconhecimento de um déficit cognitivo não implica a percepção das alterações funcionais. Além disso, é um fenômeno flutuante influenciado por fatores biológicos, psicológicos e sociais, o que torna ainda mais complexa a investigação do seu comprometimento. As diferentes hipóteses etiológicas, a operacionalização variável do conceito e a falta de instrumentos de avaliação considerados padrão-ouro impossibilitam a obtenção de resultados homogêneos sobre os fatores relacionados ao comprometimento da consciência. Consequentemente, essas dificuldades comprometem a compreensão e a investigação dos domínios envolvidos.

Estudos longitudinais ou randomizados podem contribuir com dados relevantes ao acompanharem o processo ao longo da doença e estabelecerem relações com outras variáveis, permitindo, assim, maiores esclarecimentos quanto ao impacto do comprometimento da consciência do déficit nos domínios cognitivo e funcional. Dessa forma, há necessidade de aprofundamento dos estudos na área com o objetivo de validação de instrumentos que possam fornecer dados mais consistentes de um fenômeno que pode interferir na qualidade de vida de pessoas com DA e seus cuidadores.

\section{CONTRIBUIÇÕES INDIVIDUAIS}

Tatiana Belfort - Coletou os dados e escreveu o texto.

Maria Fernanda Barroso de Sousa e Raquel Luiza

Santos - Avaliaram os resumos selecionados e contribuíram na elaboração do texto.

Marcela Lima, Bianca Torres e Raquel Dias - Avaliaram os resumos excluídos.

Marcia Cristina Nascimento Dourado - Desenhou o estudo, supervisionou a coleta de dados, revisou o texto e aprovou a versão final.

\section{CONFLITOS DE INTERESSES}

Não há.

\section{AGRADECIMENTOS}

Fundação de Apoio à Pesquisa do Estado do Rio de Janeiro (Faperj), processo no E-26/102.256/2010.

\section{REFERÊNCIAS}

1. Babinski MJ. Contribution à l'etude des troubles mentaux dans I'hemiplegie organique cerebrale (Anosognosie). Revue Neurologique. 1914;(27):845-8.

2. Morris RG, Mograbi DC. Anosognosia, autobiographical memory and self-knowledge in Alzheimer's disease. Cortex. 2012

3. Mograbi DC, Brown RG, Morris RG. Anosognosia in Alzheimer's disease - The petrified self. Conscious Cogn. 2009;18:989-1003.

4. Starkstein SE, Jorge R, Mizrahi R, Robinson RG. A diagnostic formulation for anosognosia in Alzheimer's disease. J Neurol Neurosurg Psychiatry. 2006;77:719-25.

5. Dourado MCN, Laks J, Rocha M, Soares C, Leibing A, Engelhardt E. Consciência da doença na demência: resultados preliminares em pessoas com doença de Alzheimer leve e moderada. Arq Neuropsiquiatr. 2005;63:114-8.

6. Dourado MCN, Laks J, Leibing A, Engelhardt E. Consciência da doença na demência. Rev Psiq Clín. 2006;33(6):313-21.

7. Sousa MFB, Santos RL, Brasil D, Dourado MCN. Awareness of disease in dementia of the Alzheimer type: a systematic review of longitudinal studies. J Bras Psiquiatr. 2011;60(1):50-6.

8. Clare L. Developing awareness about awareness in early-stage dementia. The role of psychosocial factor. Dementia. 2002;1(2):295-312.

9. Clare L. Managing threats to self: awareness in early stage Alzheimer's disease. Social Sci Med. 2003;57:1017-29.

10. Akai T, Haruo H, Hirofumi S, Tomohiko S, Toshihiko I. Longitudinal patterns of unawareness of memory deficits in mild Alzheimer's disease. Geriatr Gerontol Int. 2009;9:16-20.

11. Clare L, Wilson B. Longitudinal assessment of awareness in early-stage Alzheimer's disease using comparable questionnaire-base and performance-based measures: a prospective one-year follow-up study. Aging Ment Health. 2006;10(2):156-65.

12. Lamar M, Lasarev MR, Libon DJ. Determining levels of unawareness in dementia research. J Neuropsych Clin N. 2002;14(4):430-7.

13. Flashman L. Disorders of awareness in neuropsychiatric syndromes: an update. Curr Psychiatry Rep. 2002;4:346-53.

14. Clare L, Rowlands J, Bruce E, Surr C, Downs M. "I don't do like I used to do": a grounded theory approach to conceptualising awareness in people with moderate to severe dementia living in long-term care. Soc Sci Med. 2008; 66(11):2366-77.

15. Nelis SM, Clare L, Martyr A, Markova I, Roth I, Woods RT, et al. Awareness of social and emotional functioning in people with early-stage dementia and implications for carers. Aging Ment Health. 2011;15(8):961-9.

16. Amanzio M, Vase L, Leotta D, Miceli R, Palermo S, Geminiani G. Imparied awareness of deficits in Alzheimer disease: the role of everyday dysfunction. J Int Neuropsych Soc. 2013;19:63-72.

17. Sousa MFB, Santos RL, Arcoverde C, Dourado MCN, Laks J. Awareness of disease in Alzheimer's disease: preliminary results of a longitudinal study. Rev Psiq Clín. 2011;38(2):57-60.

18. Dourado MCN, Marinho V, Soares C, Engelhardt E, Laks J. Awareness of disease in dementia: development of a multidimensional rating scale. Dement Neuropsychol. 2007;1:74-80.

19. Gallo DA, Cramer AJ, Wong JT, Bennett DA. Alzheimer's disease can spare local metacognition despite global anosognosia: revisiting the confidence-accuracy relationship in episodic memory. Neuropsychologia. 2012;(50):2356-64

20. Maki Y, Amari M, Yamaguchi T, Nakaaki S, Yamaguchi H. Anosognosia: patients' distress and self-awareness of deficits in Alzheimer's disease. Am J Alzheimers Dis other Demen. 2012;27(5):339-45

21. Clare L, Nelis SM, Martyr A, Whitaker CJ, Marková IS, Roth I, et al. Longitudinal trajectories of awareness in early-stage dementia. Alzheimer Dis Assoc Disord. 2012;26(2):140-7.

22. Clare L, Nelis SM, Martyr A, Roberts J, Whitaker CJ, Marková IS, et al. The influence of psychological, social and contextual factors on the expression and measurement of awareness in early-stage dementia: testing a biopsychosocial model. Int J Geriatr Psychiatry. 2012;27:167-77.

23. Stewart G, McGeown WJ, Shanks MF, Venneri A. Anosognosia of memory impairment in Alzheimer's disease. Acta Neuropsychiatr. 2010;22:180-7.

24. Okonkwo OC, Spitznagel MB, Alosco ML, Tremont G. Associations among measures of awareness of cognitive deficits in dementia. Alzheimers Dement. 2010;6:312-8. 
25. Leicht H, Berwig M, Gertz HJ. Anosognosia in Alzheimer's disease: the role of impairment levels in assessment in insight across domains. J Int Neuropsych Soc. 2010;16:463-73

26. Ohman A, Nygard L, Kottorp A. Occupational performance and awareness of desability in mild cognitive impairment or dementia. Scand J Occup Ther. 2011;18:133-42.
27. Spitznagel MB, Tremont G, Brown LB, Gunstad J. Cognitive reserve and the relationship between depressive symptoms and awareness of deficits in dementia. J Neuropsych Clin N. 2006;18(2):186-90. 